\title{
Phase 2 study of lenvatinib in patients with advanced hepatocellular carcinoma
}

\author{
Kenji Ikeda $^{1} \cdot$ Masatoshi Kudo $^{2} \cdot$ Seiji Kawazoe $^{3} \cdot$ Yukio Osaki $^{4} \cdot$ Masafumi Ikeda $^{5} \cdot$ \\ Takuji Okusaka $^{6}$ - Toshiyuki Tamai $^{7}$ - Takuya Suzuki ${ }^{7}$ - Takashi Hisai ${ }^{7}$ • \\ Seiichi Hayato ${ }^{7} \cdot$ Kiwamu Okita $^{8} \cdot$ Hiromitsu Kumada $^{1}$
}

Received: 27 April 2016/Accepted: 6 September 2016/Published online: 4 October 2016

(c) The Author(s) 2016. This article is published with open access at Springerlink.com

\begin{abstract}
Background Lenvatinib is an oral inhibitor of vascular endothelial growth factor receptor 1-3, fibroblast growth factor receptor 1-4, platelet-derived growth factor receptor alpha, RET, and KIT. This phase 2, single-arm, open-label multicenter study evaluated lenvatinib in advanced hepatocellular carcinoma (HCC).

Methods Patients with histologically/clinically confirmed advanced HCC who did not qualify for surgical resection or local therapies received lenvatinib at a dosage of $12 \mathrm{mg}$ once daily (QD) in 28-day cycles. The primary efficacy endpoint was time to progression (TTP) per modified Response Evaluation Criteria in Solid Tumors v1.1; secondary efficacy endpoints included objective response rate
\end{abstract}

Electronic supplementary material The online version of this article (doi:10.1007/s00535-016-1263-4) contains supplementary material, which is available to authorized users.

Kenji Ikeda

ikedakenji@tora.email.ne.jp

1 Department of Hepatology, Toranomon Hospital, Toranomon 2-2-2, Minato-ku, Tokyo 105-8470, Japan

2 Department of Gastroenterology and Hepatology, Kinki University School of Medicine, Osaka, Japan

3 Department of Hepatobiliary and Pancreatology, Saga-Ken Medical Centre KOSEIKAN, Saga, Japan

4 Department of Gastroenterology and Hepatology, Osaka Red Cross Hospital, Osaka, Japan

5 Department of Hepatobiliary and Pancreatic Oncology, National Cancer Center Hospital East, Kashiwa, Japan

6 Department of Hepatobiliary and Pancreatic Oncology, National Cancer Center Hospital, Tokyo, Japan

7 Eisai Co., Ltd.,, Tokyo, Japan

8 Shunan Memorial Hospital, Yamaguchi, Japan
(ORR), disease control rate (DCR), and overall survival (OS).

Results Between July 2010 and June 2011, 46 patients received lenvatinib at sites across Japan and Korea. The median TTP, as determined by independent radiological review, was 7.4 months [95\% confidence interval (CI): 5.5-9.4]. Seventeen patients (37\%) had partial response and 19 patients $(41 \%)$ had stable disease (ORR: $37 \%$; DCR: $78 \%$ ). Median OS was 18.7 months (95\% CI: 12.7-25.1). The most common any-grade adverse events (AEs) were hypertension (76\%), palmar-plantar erythrodysesthesia syndrome (65\%), decreased appetite $(61 \%)$, and proteinuria $(61 \%)$. Dose reductions and discontinuations due to AEs occurred in $34(74 \%)$ and 10 patients (22\%), respectively. Median body weight was lower in patients with an early ( $<30$ days) dose withdrawal or reduction than in those without.

Conclusions Lenvatinib 12-mg QD showed clinical activity and acceptable toxicity profiles in patients with advanced HCC, but early dose modification was necessary in patients with lower body weight. Further development of lenvatinib in HCC should consider dose modification by body weight.

Trial registration ID www.ClinicalTrials.gov NCT009 46153.

Keywords Hepatocellular carcinoma - Lenvatinib . Tyrosine kinase inhibitor · E7080 - Vascular endothelial growth factor inhibitor

\section{Introduction}

In hepatocellular carcinoma (HCC), which accounts for 85-90 \% of primary liver cancers [1], increased expression of vascular endothelial growth factor (VEGF) levels has 
been correlated with angiogenic activity, tumor progression, and poor prognosis [2, 3].

Sorafenib is currently the only systemic VEGF-targeted therapy to have demonstrated a survival benefit in patients with advanced HCC $[4,5]$. However, the median overall survival (OS) and time to progression (TTP) with sorafenib are only $\sim 1$ year and $\sim 4$ months, respectively, with frequent dose reductions or discontinuations due to adverse events, including severe skin toxicity [6-8]. Therefore, there is still an unmet need for better therapeutic options for patients with advanced HCC. To date, phase 3 trials of several agents, including sunitinib, brivanib, and linifanib, have failed to demonstrate benefit in advanced HCC [6].

Lenvatinib - an oral, multi-tyrosine kinase receptor inhibitor of VEGF receptors 1-3, fibroblast growth factor (FGF) receptor 1-4, platelet-derived growth factor (PDGF) receptor alpha, and KIT and RET proto-oncogenes [9-11] — was approved for radioiodine-refractory differentiated thyroid cancer at a dose of $24 \mathrm{mg}$ once daily (QD) in 28-day cycles [12]. However, for the clinical development of therapeutics in HCC, re-evaluation of the starting dose of any investigational agent is recommended [13]. In a phase 1 study of lenvatinib in HCC, the maximum tolerable dose in patients with HCC and Child Pugh (CP) class A liver function was $12 \mathrm{mg}$ QD [14]. This dose also showed blood trough concentrations comparable to those with the 25-mg QD dose determined to be the maximum tolerated dose in solid tumors [15] with preliminary evidence of tumor shrinkage. Here we assessed the antitumor activity and safety of lenvatinib in this phase 2 study in patients with advanced HCC.

\section{Methods}

\section{Patients}

Patients ages 20-80 years had clinically confirmed advanced HCC with residual disease not qualifying for surgical resection or local therapies, including transarterial chemoembolization; $\geq 1$ measurable target lesion by Response Evaluation Criteria in Solid Tumors version 1.1 (RECIST v1.1); [16] 1-3 tumor lesions $>3 \mathrm{~cm}$ in diameter ( $>5 \mathrm{~cm}$ diameter if only one lesion) or four or more lesions or portal vein invasion, extrahepatic invasion; Eastern Cooperative Oncology Group performance status of 0 or 1; CP score of 5 or 6 (CP class A); platelet count $\geq 50 \times 10^{9} / \mathrm{L}$, absolute neutrophil count $\geq 1.5 \times 10^{3} / \mu \mathrm{L} ; \quad$ aspartate transaminase and alanine transaminase $\leq 5.0$ times the upper limit of normal; and serum creatinine $\leq 2.0 \mathrm{mg} / \mathrm{dL}$ or calculated creatinine clearance $\geq 40 \mathrm{~mL} / \mathrm{min}$. Patients had to have had a hepatectomy and local therapy for $\mathrm{HCC}$ at least 6 and 4 weeks, respectively, prior to study enrollment. Exclusion criteria included clinically symptomatic brain metastasis or meningeal carcinomatosis; receipt of $\geq 1$ systemic chemotherapy, including targeted therapy or transarterial infusion chemotherapy; QT-corrected interval by the Fredericia method $>500 \mathrm{~ms}$ at screening; mean blood pressure $\geq 150 / 90 \mathrm{mmHg}$; presence of a progressive central nervous system disease; or a clinically significant hemorrhagic or thrombotic event within 4 weeks prior to study enrollment.

\section{Study design and treatment}

Patients in this single-arm, open-label multicenter study of lenvatinib monotherapy for advanced HCC (NCT00946153) received daily oral administration of 12 mg lenvatinib (12-mg QD) in 28-day cycles until disease progression, unacceptable toxicity, or withdrawal of consent. Dose interruption and sequential reduction of lenvatinib (to 8- and 4-mg QD) were permitted for drug-related adverse events (AEs; see Online Resource Table S1). Once reduced, the dose could not be re-escalated. The study drug was discontinued if patient recovery time was $>2$ weeks.

This study was conducted in accordance with local laws, the Declaration of Helsinki, and International Conference on Harmonization Good Clinical Practice guidelines, and with the approval of each institutional review board. All patients provided written informed consent.

\section{Study assessments}

The primary efficacy endpoint was TTP per modified RECIST (mRECIST; modified to evaluate viable lesions) [17] by an independent radiologic review committee (IRRC). mRECIST uses viable target lesions in dynamic computed tomography and is suitable for the assessment of tyrosine kinase inhibitors in HCC [17, 18]. Secondary efficacy endpoints included objective response rate (ORR), disease control rate (DCR), and OS. Tumor response was evaluated every 8 weeks using mRECIST v1.1 by both the IRRC and study investigators. An exploratory analysis to reassess tumors using RECIST v1.1 was also performed by the IRRC. Safety was assessed by physical examinations, clinical and laboratory evaluations, vital signs, and electrocardiograms. AEs were graded according to the National Cancer Institute Common Terminology Criteria for AEs, version 3.0. A pre-dose blood sample was obtained for pharmacokinetic (PK) assessment on days 1, 8, 15, and 22 of cycle 1 , and day 1 of cycles 2 and 3, using a validated liquid chromatography-tandem mass spectrometry method [19]. 


\section{Statistical considerations}

Time-to-event endpoints, including TTP and OS, were summarized using Kaplan-Meier estimates. The sample size of 46 patients was based on an $80 \%$ probability that the lower limit of the two-sided $90 \%$ confidence interval (CI) of median TTP would exceed the threshold of 2.7 months (based on the Sorafenib HCC Assessment Randomized Protocol trial [20]), with an expected median TTP of 4.1 months for lenvatinib. Sample size determination also assumed exponential distribution for TTP, 12 months of enrollment, 6 months of follow-up, and a $10 \%$ patient exclusion/dropout rate. An interim evaluation was conducted when 21-23 patients became evaluable for the 2-month tumor assessment. If the number of patients with progressive disease within 2 months was $\geq 10$ ( $\geq 80 \%$ Bayesian posterior probability for proportion of patients with progressive disease at 2 months $\geq 35 \%$ ), then study discontinuation would be considered due to futility. Follow-up was continued until final analysis was performed when $67 \%$ of patients had died.

\section{Results}

\section{Patient characteristics}

Overall, 46 patients were enrolled and received lenvatinib at 14 sites across Japan and Korea between July 2010 and June 2011. All patients were included in the safety and efficacy analyses. Patient demographics and baseline characteristics are listed in Table 1.

\section{Efficacy}

Median TTP was 7.4 months (95\% CI: 5.5-9.4) as assessed by IRRC per mRECIST (Fig. 1a). Median TTP was 12.8 months (95\% CI: 7.2-14.7) by investigator assessment. Seventeen patients $(37 \%)$ achieved a partial response and 19 patients $(41 \%)$ had stable disease $\geq 8$ weeks, with a DCR of $78 \%$ by IRRC (Table 2). Outcomes using RECIST v1.1 criteria are also provided in Table 2. Median OS was 18.7 months (95\% CI: 12.7-25.1; Fig. 1b).

Tumor reduction of target lesions, assessed by IRRC, occurred in $80 \%$ of patients (Fig. 2a-c). Subgroup analyses indicated that lenvatinib clinical activity was maintained regardless of tumor status (with or without extrahepatic spread or portal vein invasion), type of underlying hepatitis [hepatitis B virus (HBV) or hepatitis C virus], receipt of previous chemotherapy, or alpha-fetoprotein levels (AFP; $<200 \mathrm{ng} / \mathrm{mL}$ or $\geq 200 \mathrm{ng} / \mathrm{mL}$; see
Table 1 Patient demographics and baseline characteristics

\begin{tabular}{|c|c|}
\hline Characteristic & Patients $(N=46)$ \\
\hline Median age, years (range) & $66.5(37-80)$ \\
\hline \multicolumn{2}{|l|}{ Sex, $n(\%)$} \\
\hline Female & $13(28.3)$ \\
\hline Male & $33(71.7)$ \\
\hline \multicolumn{2}{|l|}{ Region, $n(\%)$} \\
\hline Japan & $43(93.5)$ \\
\hline South Korea & $3(6.5)$ \\
\hline Median weight, kg (range) & $56.7(42.8-85.5)$ \\
\hline \multicolumn{2}{|l|}{ ECOG PS, $n(\%)$} \\
\hline 0 & $38(82.6)$ \\
\hline 1 & $8(17.4)$ \\
\hline \multicolumn{2}{|l|}{ Child Pugh Class, $n(\%)$} \\
\hline A & $45(97.8)$ \\
\hline B & $1(2.2)$ \\
\hline \multicolumn{2}{|l|}{ BCLC staging, $n(\%)$} \\
\hline $\mathrm{B}$ & 19 (41.3) \\
\hline $\mathrm{C}$ & $27(58.7)$ \\
\hline \multicolumn{2}{|l|}{ Portal vein invasion, $n(\%)$} \\
\hline Yes & $5(10.8)$ \\
\hline No & $41(89.1)$ \\
\hline \multicolumn{2}{|l|}{ Extrahepatic metastasis, $n(\%)$} \\
\hline Yes & $21(45.7)$ \\
\hline No & $25(54.3)$ \\
\hline \multicolumn{2}{|l|}{ Cause of HCC, $n(\%)$} \\
\hline Hepatitis B & $15(32.6)$ \\
\hline Hepatitis C & $27(58.7)$ \\
\hline Alcohol & $2(4.3)$ \\
\hline Non-alcohol-related fatty liver disease & $1(2.2)$ \\
\hline Unknown & $2(4.3)$ \\
\hline \multicolumn{2}{|l|}{ AFP value at baseline ${ }^{a}$} \\
\hline$<200 \mathrm{ng} / \mathrm{mL}$ & $27(57.7)$ \\
\hline$\geq 200 \mathrm{ng} / \mathrm{mL}$ & $18(39.1)$ \\
\hline \multicolumn{2}{|l|}{ Prior surgery for HCC, $n(\%)$} \\
\hline No & $27(58.7)$ \\
\hline Yes & $19(41.3)$ \\
\hline \multicolumn{2}{|l|}{ Prior local therapy, $n(\%)$} \\
\hline No & $4(8.7)$ \\
\hline Yes & $42(91.3)$ \\
\hline RFA & $32(69.6)$ \\
\hline PEI & $12(26.1)$ \\
\hline TACE & $39(84.8)$ \\
\hline TAE & $3(6.5)$ \\
\hline \multicolumn{2}{|l|}{ Prior chemotherapy, $n(\%)$} \\
\hline Sorafenib & $6(13.0)$ \\
\hline Other systemic chemotherapy & $5(10.9)$ \\
\hline Hepatic intra-arterial chemotherapy & $5(10.9)$ \\
\hline
\end{tabular}

BCLC Barcelona Clinic Liver Cancer, ECOG-PS Eastern Cooperative Oncology Group Performance Status, $H C C$ hepatocellular carcinoma, $A F P$ alpha-fetoprotein, $P E I$ percutaneous ethanol injection, $R F A$ radiofrequency ablation, TACE transcatheter arterial chemoembolization, $T A E$ transarterial embolization

a AFP data were unavailable for one patient 


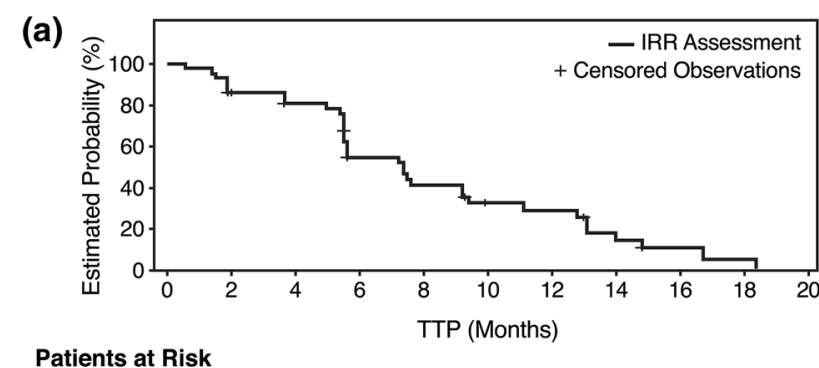

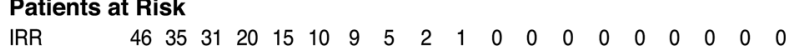

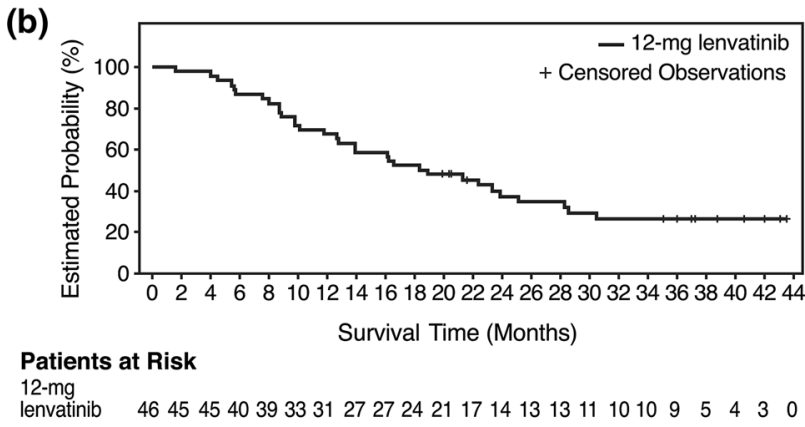

Fig. 1 Kaplan-Meier estimates of a TTP and b OS. Median TTP was 7.4 months as assessed by an IRRC comprising four independent radiologists. Median OS was 18.7 months. IRRC independent radiologic review committee, TTP time to progression

Online Resource Table S2). On the other hand, AFP levels may affect the prognosis for patients with HCC (Online Resource Figure S1).

\section{Safety}

Median and mean durations of lenvatinib treatment were 7.3 and 9.0 months, respectively. All 46 patients experienced at least one AE. The most common any-grade AEs (Table 3) were hypertension (76\%), palmar-plantar erythrodysesthesia syndrome (PPES; $65 \%$ ), decreased appetite $(61 \%)$, and proteinuria $(61 \%)$. The incidence of serious AEs (SAEs) was $48 \%$, and the most frequently reported SAE was hepatic encephalopathy (11\%). No treatment-related deaths were reported. Two patients died within 30 days of receiving their last dose of lenvatinibone from pneumonia and one from liver tumor rupture.

AEs were generally manageable with dose modifications. Lenvatinib dose reductions due to AEs occurred in 34 patients $(74 \%)$. Ten patients $(22 \%)$ discontinued study treatment due to toxicity. The most frequently reported AE leading to study drug withdrawal was proteinuria $(11 \%)$. Twenty-two patients (48\%) experienced AEs leading to dose withdrawal or dose reduction $<30$ days after starting lenvatinib. In an exploratory analysis of differences in baseline characteristics between patients who did and did not require an early dose withdrawal or reduction, body weight and minimum concentration of lenvatinib $\left(\mathrm{C}_{\text {trough }}\right)$ were identified as potential differentiators (Fig. 3). Median body weight was lower for patients who experienced an early dose withdrawal or reduction $(54.1 \mathrm{~kg})$ than for those who did not (67.6 kg) (Fig. 3a). The median $\mathrm{C}_{\text {trough }}$ values on cycle 1 day 15 (C1D15) in patients with and without dose modifications were 62.4 and $33.9 \mathrm{ng} / \mathrm{mL}$, respectively (Fig. 3b). The Spearman correlation coefficient between body weight and lenvatinib $\mathrm{C}_{\text {trough }}$ at C1D15 was -0.64 .

\section{Discussion}

Multi-tyrosine kinase inhibitors have shown limited success in advanced HCC, with reported TTP ranging from 2.8 to 5.4 months and ORR from 6.9 to $9 \%$ [4, 5, 7, 21, 22]. In this study, lenvatinib 12-mg QD showed promising clinical activity in patients with advanced HCC, with a median TTP of 7.4 months as assessed by IRRC (12.8 months by investigator). Lenvatinib also demonstrated tumor shrinkage in $80 \%$ of patients, with a response rate of $37 \%$ per mRECIST and $24 \%$ per conventional RECIST.

Although progression-free survival is the preferred OS surrogate endpoint in most solid tumor trials, it is a particularly unreliable endpoint in HCC studies, because death from the natural history of cirrhosis may confound results;

Table 2 Tumor responses

\begin{tabular}{lccc}
\hline Response category & $\begin{array}{l}\text { Investigator assessment } \\
(\mathrm{mRECIST}), n=46\end{array}$ & $\begin{array}{l}\text { IRRC assessment } \\
\text { (mRECIST), } n=46\end{array}$ & $\begin{array}{l}\text { IRRC assessment } \\
\text { (RECIST 1.1), } n=46\end{array}$ \\
\hline Best response, $n(\%)$ & & & $0(0)$ \\
$\quad$ Complete response & $0(0)$ & $0(0)$ & $11(24)$ \\
Partial response & $17(37)$ & $17(37)$ & $25(54)$ \\
Stable disease & $21(46)$ & $19(41)$ & $6(13)$ \\
Progressive disease & $5(11)$ & $6(13)$ & $4(9)$ \\
Not evaluable & $3(7)$ & $4(9)$ & $11(24)$ \\
Objective response rate, $n(\%)$ & $17(37)$ & $17(37)$ & $36(78)$ \\
Disease control rate, $n(\%)$ & $38(83)$ & $36(78)$ &
\end{tabular}

IRRC independent radiologic review committee, mRECIST modified response evaluation criteria in solid tumors 
Fig. 2 a Waterfall plot of changes in tumor size by IRRC assessment. One patient was excluded from the plot due to lack of IRRC assessment of target legion at baseline. The patient marked with an asterisk showed a best overall response of SD. b Representative liver lesion of HCC at baseline on arterial phase CT.

c Representative liver lesion on arterial phase CT after 1 year of lenvatinib treatment. $C T$ computed tomography, $H C C$ hepatocellular carcinoma, IRRC independent radiologic review committee, $P D$ progressive disease, $P R$ partial response, $S D$ stable disease, $N E$ not evaluable

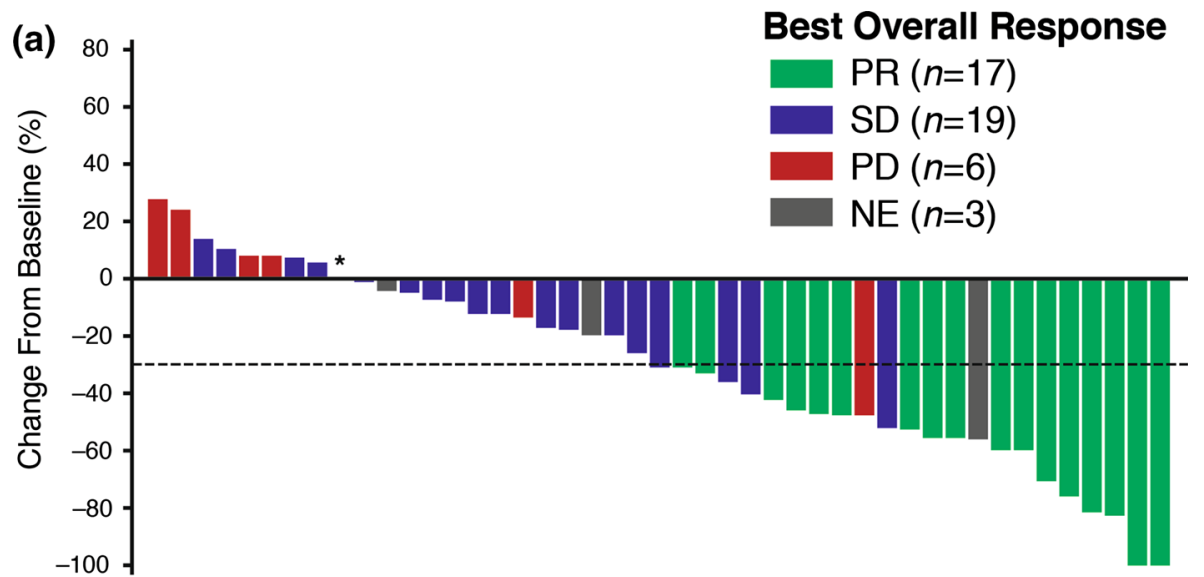

(b)

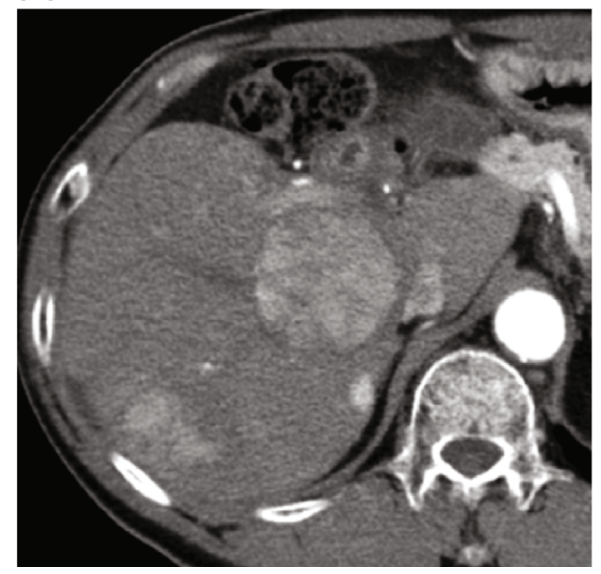

(c)

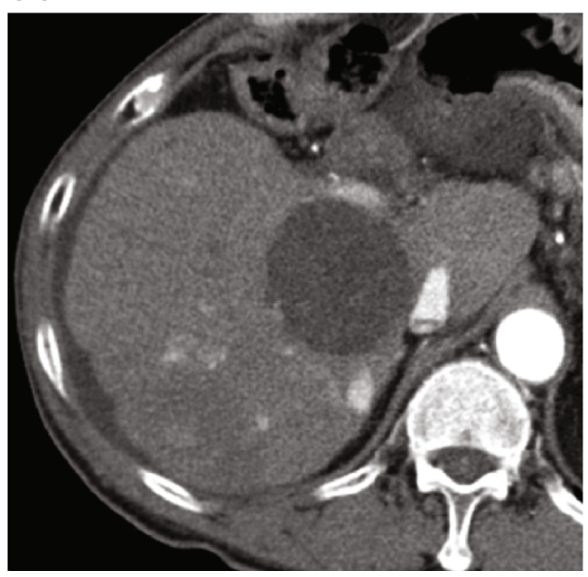

TTP is therefore the recommended endpoint for early-stage trials of HCC [13]. In HCC, response rates derived from mRECIST have been shown to better correlate with OS than those from conventional RECIST [18]. Notably, despite a similarity in best overall responses between investigator and independent assessments, the median TTP was substantially different, suggesting a bias by the investigators in determining the timing of disease progression, ostensibly so that their patients could continue therapy.

The median OS in this study was 18.7 months. Although subgroup analyses indicated that median TTP was comparable regardless of baseline AFP levels, OS was longer in the $61 \%$ of patients with lower vs. higher AFP levels (23.5 vs. 13.3 months, respectively). Therefore, it is possible that the long median OS observed in this study may have been driven by those patients with lower baseline AFP levels, because elevated AFP levels are associated with an increased mortality rate in HCC [23]. Other risk factors examined included extrahepatic spread and HBV. In this study, lenvatinib activity was observed even in these patients with poor prognoses; however, this conclusion is limited by the small numbers of patients in each subgroup. Another limitation is the single-arm design of the study, and the possibility that results may be influenced by patient selection. However, we note that patient characteristics in this study were typical of the population of patients with HCC who required sorafenib therapy in Japan [8, 24].

The most common AEs in this study included hypertension, PPES, decreased appetite, proteinuria, and fatigue, which are well-known class effects of VEGF-targeted therapies and are consistent with the known safety profile of lenvatinib. Although the incidence of grade 3 hypertension was high $(54 \%)$, this included patients whose blood pressure was controlled by two or more antihypertensive drugs. No patient required a dose modification or discontinuation due to hypertension; therefore, it was considered to be manageable. Although $65 \%$ of patients experienced PPES, the incidence of grade 3 PPES was only $9 \%$. The incidence of grade 3 or 4 thrombocytopenia was also high (22\%); however, in all but one patient who discontinued lenvatinib, thrombocytopenia was controllable with dose modifications. There was no report of grade 3 or higher bleeding related to the study drug. Although hepatic encephalopathy was the most common SAE (five patients) in this study, all five patients also had constipation, and three had dehydration-known risk factors for 
Table 3 Common adverse events occurring in $\geq 20 \%$ of patients

\begin{tabular}{|c|c|c|c|}
\hline Adverse event & $\begin{array}{l}\text { Any } \\
\text { grade, } \\
n=46\end{array}$ & $\begin{array}{l}\text { Grade } 3, \\
n=46\end{array}$ & $\begin{array}{l}\text { Grade 4, } \\
n=46\end{array}$ \\
\hline Hypertension & $35(76.1)$ & $25(54.3)$ & 0 \\
\hline $\begin{array}{l}\text { Palmar-plantar } \\
\text { erythrodysesthesia } \\
\text { syndrome }\end{array}$ & $30(65.2)$ & $4(8.7)$ & 0 \\
\hline Decreased appetite & $28(60.9)$ & $1(2.2)$ & 0 \\
\hline Proteinuria & $28(60.9)$ & 9 (19.6) & 0 \\
\hline Fatigue & $25(54.3)$ & 0 & 0 \\
\hline Diarrhea & $20(43.5)$ & $6(13.0)$ & 0 \\
\hline Constipation & $19(41.3)$ & 0 & 0 \\
\hline Nausea & $17(37.0)$ & $1(2.2)$ & 0 \\
\hline Dysphonia & $17(37.0)$ & 0 & 0 \\
\hline Thrombocytopenia & $16(34.8)$ & 9 (19.6) & $1(2.2)$ \\
\hline Peripheral edema & $16(34.8)$ & 0 & 0 \\
\hline Decreased weight & $14(30.4)$ & $2(4.3)$ & 0 \\
\hline Neutropenia & $13(28.3)$ & $2(4.3)$ & 0 \\
\hline Nasopharyngitis & $13(28.3)$ & 0 & 0 \\
\hline Rash & $13(28.3)$ & 0 & 0 \\
\hline $\begin{array}{l}\text { Increased blood thyroid- } \\
\text { stimulating hormone level }\end{array}$ & $12(26.1)$ & 0 & 0 \\
\hline Back pain & $11(23.9)$ & 0 & 0 \\
\hline Stomatitis & $11(23.9)$ & 0 & 0 \\
\hline Vomiting & $11(23.9)$ & $1(2.2)$ & 0 \\
\hline Pyrexia & $10(21.7)$ & 0 & 0 \\
\hline Hypothyroidism & $10(21.7)$ & 0 & 0 \\
\hline Insomnia & $10(21.7)$ & 0 & 0 \\
\hline
\end{tabular}

hepatic encephalopathy [25]. These events were managed with dose modifications as well as treatment of constipation and dehydration.

Dose reduction occurred frequently and early in the course of treatment. Because $74 \%$ of patients required a dose reduction due to AEs, we examined possible risk factors for the development of intolerable toxicities. The influence of body weight on the pharmacodynamics of antiangiogenic agents and resultant toxicity patterns is still uncertain [26]. Additionally, increased lenvatinib exposure was found in patients with severe hepatic impairment [27]. It is possible that in patients with HCC, who typically have impaired hepatic function, lenvatinib PK is more affected by body weight than in healthy individuals or patients with other cancers. Furthermore, careful evaluation of the balance between efficacy and toxicity is especially important in studies of patients with HCC, because high toxicity is a common reason for failure of phase 3 clinical trials in this therapeutic area [6]. Therefore, although drug-related AEs were manageable with dose modifications, and there were no drug-related deaths in this study, lenvatinib exposure was observed to be influenced by body weight in patients
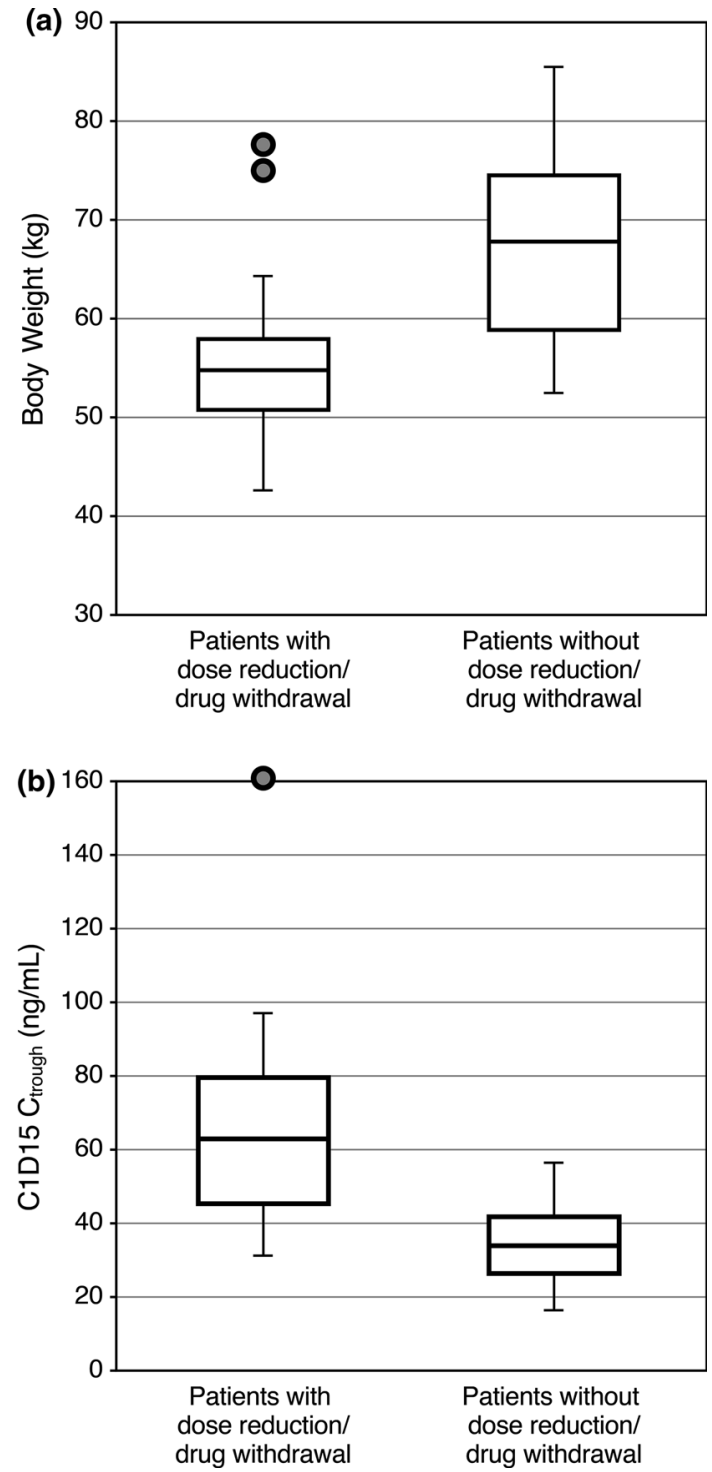

Fig. 3 a Boxplot of body weight for patients with vs. without adverse events that led to dose reduction or dose withdrawal within 30 days of lenvatinib treatment initiation. b Boxplot of lenvatinib $\mathrm{C}_{\text {trough }}$ level 15 days after lenvatinib treatment initiation for patients with vs. without adverse events that led to dose reduction or withdrawal within 30 days. C1D15 cycle 1 day $15, C_{\text {trough }}$ minimum concentration of lenvatinib

with HCC, and adjustment of the starting dose of lenvatinib by body weight in further clinical development of lenvatinib in HCC is recommended.

In conclusion, the administration of lenvatinib 12-mg QD showed clinical activity and acceptable toxicity profiles in patients with advanced HCC, although early dose modification was necessary for the management of toxicities in patients with lower body weight. Based on these findings, a phase 3 study of lenvatinib in HCC is underway, with planned doses of $8 \mathrm{mg}$ in patients with lower body weight $(<60 \mathrm{~kg})$ and $12 \mathrm{mg}$ in those with higher body weight ( $\geq 60 \mathrm{~kg}$; NCT01761266) [28]. 
Acknowledgments We thank all of the patients who participated in this study and their families, as well as all investigators, physicians, nurses, and clinical research coordinators who helped with the study, including the Independent Radiological Review Committee: Drs. Osamu Matsui (Kanazawa University Hospital), Kazuhiko Ueda (Shinshu University Hospital), Akihiro Tanimoto (Keio University Hospital), and Tatsuya Gomi (Toho University Ohashi Medical Center). We also thank the Efficacy and Safety Evaluation Committee: Drs. Junji Furuse (Kyorin University School of Medicine), Kazuhiko Koike (The University Tokyo), and Shigeki Arii (Hamamatsu Rousai Hospital). Editorial assistance was provided by Oxford PharmaGenesis Inc., and funded by Eisai Inc. The work was supported by Eisai Co., Ltd., Japan.

\section{Compliance with ethical standards}

Conflict of interest Dr. K. Ikeda reports honoraria from Eisai, Dainippon, Sumitomo Pharmaceutical Co., and Olympus, and served in a consulting/advisory role for Eisai during the conduct of study. Dr. Kudo reports honoraria from Bayer, Eisai, MSD, Bristol Myers Squibb, Daiichi, and Sankyo, and served in a consulting/advisory role for Eisai, Bristol Myers Squibb, and Eli Lilly. Dr. Kawazoe has nothing to disclose. Dr. Osaki has nothing to disclose. Dr. M. Ikeda reports honoraria from Novartis, Bayer Yakuhin, Bristol Myers Squibb, Abbott Japan, Taiho Pharmaceutical, Eli Lilly Japan, Kowa, OncoTherapy Science, Nippon Kayaku, and Daiichi Sankyo; received research funding from Bayer Yakuhin, Novartis, Merck Serono, Kyowa Hakko Kirin, Yakult, Taiho Pharmaceutical, Eli Lilly Japan, Otsuka Pharmaceutical, OncoTherapy Science, Boehringer Ingelheim, Kowa, Ono Pharmaceutical, Eisai, AstraZeneca, Pfizer Japan, Glaxo Smith Kline, and Zeria Pharmaceutical; and reports travel, accommodation, and other expense reimbursement from Bayer Yakuhin and Eisai. Dr. Okusaka reports honoraria from Chugai Pharmaceutical Co., Ltd, Pfizer Japan Inc., Novartis Pharmaceutical K.K., Taiho Pharmaceutical Co., Ltd., Merck Serono Co., Ltd., Eli Lilly Japan K.K., Dainippon Sumitomo Pharmaceutical Co., Ltd, Eisai Co., Ltd, and Bayer Yakuhin Ltd.; served in a consulting/advisory role for Eli Lilly Japan K.K., Yakult Honsha Co., Ltd., Amgen, Dainippon Sumitomo Pharmaceutical Co., Ltd., Taiho Pharmaceutical Col, Ltd., OncoTherapy Science, Inc., Nobelpharma Co., Ltd., Nippon Boehringer Ingelheim Co., Ltd., Nano Carrier Co., Ltd., Novartis Pharmaceutical K.K., and Zeria Pharmaceutical Co., Ltd.; and received research funding from Chugai Pharmaceutical Co., Ltd., Eli Lilly Japan K.K., Eisai Co., Ltd., Novartis Pharmaceutical K.K., Shizuoka Industry, Takeda Bio Development Center Limited, Yakult Honsha Co., Ltd., OncoTherapy Science, Inc., Otsuka Pharmaceutical Co., Ltd., Taiho Pharmaceutical Co., Ltd., Sceti Medical Laboratory K.K., Nippon Boehringer Ingelheim Co., Ltd., Kowa Company, Ltd., Kyowa Hakko Kirin Co., Ltd, Merck Serono Co., Ltd, Ono Pharmaceutical Co., Ltd. Bayer Yakuhin Ltd., Pfizer Japan Inc., AstraZeneca K.K., and Dainippon Sumitomo Pharmaceutical Co., Ltd. T. Tamai reports employment and stock/other ownership with Eisai Co., Ltd. T. Suzuki reports employment with Eisai Co., Ltd. T. Hisai has nothing to disclose. S. Hayato reports employment with Eisai Co., Ltd. Dr. Okita reports honoraria from Kowa and Eisai Co., Ltd. Dr. Kumada reports honoraria from, and served in a consulting/advisory role for, Eisai Co., Ltd.

Open Access This article is distributed under the terms of the Creative Commons Attribution 4.0 International License (http://crea tivecommons.org/licenses/by/4.0/), which permits unrestricted use, distribution, and reproduction in any medium, provided you give appropriate credit to the original author(s) and the source, provide a link to the Creative Commons license, and indicate if changes were made.

\section{References}

1. El-Serag HB, Rudolph KL. Hepatocellular carcinoma: epidemiology and molecular carcinogenesis. Gastroenterology. 2007;132:2557-76.

2. Poon RT, Ho JW, Tong CS, Lau C, Ng IO, Fan ST. Prognostic significance of serum vascular endothelial growth factor and endostatin in patients with hepatocellular carcinoma. Br J Surg. 2004;91:1354-60.

3. Bertino G, Demma S, Ardiri A, et al. Hepatocellular carcinoma: novel molecular targets in carcinogenesis for future therapies. Biomed Res Int. 2014;2014:203693.

4. Llovet JM, Ricci S, Mazzaferro V, et al. Sorafenib in advanced hepatocellular carcinoma. N Engl J Med. 2008;359:378-90.

5. Cheng AL, Kang YK, Chen Z, et al. Efficacy and safety of sorafenib in patients in the Asia-Pacific region with advanced hepatocellular carcinoma: a phase III randomised, double-blind, placebo-controlled trial. Lancet Oncol. 2009;10:25-34.

6. Llovet JM, Hernandez-Gea V. Hepatocellular carcinoma: reasons for phase III failure and novel perspectives on trial design. Clin Cancer Res. 2014;20:2072-9.

7. Cheng AL, Kang YK, Lin DY, et al. Sunitinib versus sorafenib in advanced hepatocellular cancer: results of a randomized phase III trial. J Clin Oncol. 2013;31:4067-75.

8. Ogasawara S, Kanai F, Obi S, et al. Safety and tolerance of sorafenib in Japanese patients with advanced hepatocellular carcinoma. Hepatol Int. 2011;5:850-6.

9. Matsui J, Funahashi Y, Uenaka T, Watanabe T, Tsuruoka A, Asada M. Multi-kinase inhibitor E7080 suppresses lymph node and lung metastases of human mammary breast tumor MDA-MB231 via inhibition of vascular endothelial growth factor-receptor (VEGF-R) 2 and VEGF-R3 kinase. Clin Cancer Res. 2008;14:5459-65.

10. Tohyama O, Matsui J, Kodama K, et al. Antitumor activity of lenvatinib (e7080): an angiogenesis inhibitor that targets multiple receptor tyrosine kinases in preclinical human thyroid cancer models. J Thyroid Res. 2014;2014:638747.

11. Yamamoto Y, Matsui J, Matsushima T, et al. Lenvatinib, an angiogenesis inhibitor targeting VEGFR/FGFR, shows broad antitumor activity in human tumor xenograft models associated with microvessel density and pericyte coverage. Vasc Cell. 2014;6:18.

12. Schlumberger M, Tahara M, Wirth $\mathrm{LJ}$, et al. Lenvatinib versus placebo in radioiodine-refractory thyroid cancer. N Engl J Med. 2015;372:621-30.

13. Llovet JM, Di Bisceglie AM, Bruix J, et al. Design and endpoints of clinical trials in hepatocellular carcinoma. J Natl Cancer Inst. 2008;100:698-711.

14. Ikeda M, Okusaka T, Mitsunaga S, et al. Safety and pharmacokinetics of lenvatinib in patients with advanced hepatocellular carcinoma. Clin Cancer Res. 2016;22:1385-94.

15. Boss DS, Glen H, Beijnen JH, et al. A phase I study of E7080, a multitargeted tyrosine kinase inhibitor, in patients with advanced solid tumours. Br J Cancer. 2012;106:1598-604.

16. Eisenhauer EA, Therasse P, Bogaerts J, et al. New response evaluation criteria in solid tumours: revised RECIST guideline (version 1.1). Eur J Cancer. 2009;45:228-47.

17. Lencioni R, Llovet JM. Modified RECIST (mRECIST) assessment for hepatocellular carcinoma. Semin Liver Dis. 2010;30:52-60.

18. Edeline J, Boucher E, Rolland Y, et al. Comparison of tumor response by Response Evaluation Criteria in Solid Tumors (RECIST) and modified RECIST in patients treated with sorafenib for hepatocellular carcinoma. Cancer. 2012;118:147-56. 
19. Mano Y, Kusano K. A validated LC-MS/MS method of total and unbound lenvatinib quantification in human serum for protein binding studies by equilibrium dialysis. J Pharm Biomed Anal. 2015;114:82-7.

20. Sherman M, Mazzaferro V, Amadori D, et al. Efficacy and safety of sorafenib in patients with advanced hepatocellular carcinoma and vascular invasion or extrahepatic spread: a subanalysis from the SHARP trial [abstract]. J Clin Oncol. 26:15S. 2008. (abstr 4584).

21. Johnson PJ, Qin S, Park JW, et al. Brivanib versus sorafenib as first-line therapy in patients with unresectable, advanced hepatocellular carcinoma: results from the randomized phase III BRISK-FL study. J Clin Oncol. 2013;31:3517-24.

22. Cainap C, Qin S, Huang WT, et al. Linifanib versus sorafenib in patients with advanced hepatocellular carcinoma: results of a randomized phase III trial. J Clin Oncol. 2015;33:172-9.

23. Li P, Wang SS, Liu H, et al. Elevated serum alpha fetoprotein levels promote pathological progression of hepatocellular carcinoma. World J Gastroenterol. 2011;17:4563-71.
24. Takeda H, Nishikawa H, Osaki Y, et al. Clinical features associated with radiological response to sorafenib in unresectable hepatocellular carcinoma: a large multicenter study in Japan. Liver Int. 2015;35:1581-9.

25. Wright G, Chattree A, Jalan R. Management of hepatic encephalopathy. Int J Hepatol. 2011;2011:841407.

26. Verheul HM, Pinedo HM. Possible molecular mechanisms involved in the toxicity of angiogenesis inhibition. Nat Rev Cancer. 2007;7:475-85.

27. Shumaker R, Aluri J, Fan J, Martinez G, Pentikis H, Ren M. Influence of hepatic impairment on lenvatinib pharmacokinetics following single-dose oral administration. J Clin Pharmacol. 2015;55:317-27.

28. Finn RS, Cheng AL, Ikeda K, et al. A multicenter, open-label, phase 3 trial to compare the efficacy and safety of lenvatinib (E7080) verus sorafenib in first-line treatment of subjects with unresectable hepatocellular carcinoma [abstract]. J Clin Oncol.32:5s. 2014. (abstr TPS4153). 